\title{
Saturação por Bases e Doses de Fósforo na Produção de Mudas de Ipê Rosa (Handroanthus impetiginosa)
}

\section{Base Saturation and Doses of Phosphorus in the Pink Ipe (Handroanthus impetiginosa) Seedlings Production}

\author{
Cristiane Ramos Vieira*a; Rayza Samara de Assis Carneiro ${ }^{a}$ \\ anniversidade de Cuiabá, Programa de Pós-Graduação Stricto Sensu em Ciências Ambientais. MT, Brasil. \\ *E-mail: cris00986@hotmail.com.
}

\begin{abstract}
Resumo
O ipê rosa (Handroanthus impetiginosa) é uma espécie arbórea nativa do Brasil usada na ornamentação e recomposição florestal. Para garantir que mudas de qualidade sejam utilizadas em qualquer finalidade, para a qual se emprega essa espécie, deve-se conhecer mais a respeito das suas exigências nutricionais. Diante disso, um experimento foi realizado com o objetivo de avaliar a saturação por bases e diferentes níveis de fósforo $(\mathrm{P})$ na produção de mudas do ipê rosa. $\mathrm{O}$ experimento foi conduzido em delineamento inteiramente casualizado com nove tratamentos e cinco repetições: T0 (100\% substrato comercial); T1 (V50\% de saturação por bases); T2 (V60\% de saturação por bases); T3 (V50\% de saturação por bases $+50 \mathrm{~kg} \mathrm{ha}^{-1}$ de $\left.\mathrm{P}_{2} \mathrm{O}_{5}\right)$; T4 (V50\% de saturação por bases $+100 \mathrm{~kg} \mathrm{ha}^{-1}$ de $\left.\mathrm{P}_{2} \mathrm{O}_{5}\right)$; T5 (V50\% de saturação por bases +150 $\mathrm{kg} \mathrm{ha}^{-1}$ de $\left.\mathrm{P}_{2} \mathrm{O}_{5}\right)$; T6 (V60\% de saturação por bases $+50 \mathrm{~kg} \mathrm{ha}^{-1}$ de $\left.\mathrm{P}_{2} \mathrm{O}_{5}\right)$; T7 (V60\% de saturação por bases $+100 \mathrm{~kg}$ ha ${ }^{-1}$ de $\left.\mathrm{P}_{2} \mathrm{O}_{5}\right)$; T8 $(\mathrm{V} 60 \%$ de saturação por bases $+150 \mathrm{~kg} \mathrm{ha}^{-1} \mathrm{de}_{\mathrm{P}_{2}} \mathrm{O}_{5}$ ). As características morfológicas foram avaliadas ao final de 90 dias. A saturação por bases e a adubação fosfatada não influenciaram no crescimento em altura e em diâmetro das mudas de H. impetiginosa, porém, influenciaram na produção de massa seca. Dessa forma, recomenda-se, para a produção de mudas de H. impetiginosa, a preparação do substrato sendo: solo com

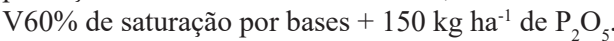

Palavras-chave: Calagem. Fosfatagem. Nutrição de Plantas. Viveiro.

\begin{abstract}
The pink ipe (Handroanthus impetiginosa) is a tree species native to Brazil that is used in forest ornamentation and restoration. To ensure that quality seedlings are used for any purpose, for which this species is used, it is necessary to know more about nutritional requirements. Therefore, an experiment was developed to evaluate base saturation and different phosphorus $(P)$ levels in the pink ipe seedlings production. The experiment was carried out in a completely randomized design with nine treatments and five replications: T0 (100\% commercial substrate); $T 1$ (V50\% of base saturation); T2 (V60\% of base saturation); T3 (V50\% of base saturation $+50 \mathrm{~kg} \mathrm{ha-1}$ of $\mathrm{P}_{2} \mathrm{O}_{5}$ ); T4 (V50\% of base saturation $+100 \mathrm{~kg} \mathrm{ha} a_{-1}$ of $\left.\mathrm{P}_{2} \mathrm{O}_{5}\right) ; T 5\left(\mathrm{~V} 50 \%\right.$ of base saturation $+150 \mathrm{~kg} \mathrm{ha}-1$ of $\left.\mathrm{P}_{2} \mathrm{O}_{5}\right) ; \mathrm{T6}\left(\mathrm{V} 60 \%\right.$ of base saturation $+50 \mathrm{~kg} \mathrm{ha} a^{-1}$ of $\left.\mathrm{P}_{2} \mathrm{O}_{5}\right) ; \mathrm{T} 7(\mathrm{~V} 60 \%$ of base saturation $+100 \mathrm{~kg} \mathrm{ha}^{-1}$ of $\left.\mathrm{P}_{2} \mathrm{O}_{5}\right) ; \mathrm{T} 8\left(\mathrm{~V} 60 \%\right.$ of base saturation $\left.+150 \mathrm{~kg} \mathrm{ha}^{-1} \mathrm{P}_{2} \mathrm{O}_{5}\right)$. Morphological characteristics of the plants were evaluated after 90 days. Base saturation and phosphate fertilization did not influence H. impetiginosa seedling height and diameter growth but influenced dry mass production. Thus, for the H. impetiginosa seedlings production, substrate preparation: soil with V60\% of base saturation $+150 \mathrm{~kg} \mathrm{ha}^{-1}$ of $\mathrm{P}_{2} \mathrm{O}_{5}$ is recommended.
\end{abstract}

Keywords: Liming. Phosphating. Plant Nutrition. Nursery.

\section{Introdução}

Os solos brasileiros, especialmente os de Cerrado, são na sua maioria, naturalmente ácidos, tendo baixa disponibilidade de nutrientes (SILVA et al., 2011; COSTA FILHO et al., 2013) necessários para o crescimento das plantas. Isso dificulta a utilização desse solo, como substrato único para a produção de mudas em viveiros florestais, sem a adição de um material que tenha capacidade nutritiva maior, ou mesmo a adição de adubos minerais comerciais.

Segundo Freitas et al. (2017a), entre os substratos utilizados para a produção de mudas de espécies arbóreas nativas, destaca-se a terra de subsolo, que ainda é utilizada pelos viveiros. Todavia, a maioria dos solos brasileiros apresenta elevada acidez e baixa disponibilidade de nutrientes em condições naturais, sendo o principal deles, o fósforo (P). Daí a importância da adubação durante a produção das mudas no viveiro. Dutra et al. (2015) destacaram que uma das formas de se produzir mudas de boa qualidade e que tenham chance de sobrevivência após o transplantio, é através de uma nutrição equilibrada, utilizando-se das adubações minerais. Dessa forma, o sucesso na utilização de espécies florestais nativas, depende do conhecimento dos seus requerimentos nutricionais, visando aperfeiçoar o sistema de produção de mudas e, consequentemente, aumentar o seu potencial de sobrevivência e crescimento após o plantio no campo.

O ipê rosa (Handroanthus impetiginosa antigo Tabebuia impetiginosa), espécie arbórea nativa do Brasil, que pertence à família Bignoniaceae, tornou-se ameaçado de extinção 
devido a supressão da vegetação natural para a implantação de cultivos agrícolas e da pastagem (SILVA et al., 2004). De acordo com Gemaque et al. (2002) esta espécie foi intensamente explorada nas regiões de sua ocorrência natural, restando poucas árvores isoladas. Por esses fatos, ela tem sido indicada nos trabalhos de restauração de ecossistemas florestais e de paisagismo.

Portanto, produzir mudas dessa espécie para que se faça a recomposição das áreas nativas se faz necessário. No entanto, essa produção requer informações cada vez mais específicas a respeito das etapas do processo. Uma dessas etapas é a complementação do substrato com os nutrientes em quantidades necessárias para o crescimento das plantas. Uma das intervenções que se pode fazer para melhorar as condições de crescimento dessas plantas, na sua fase inicial de desenvolvimento, é a calagem. Outra intervenção é a adubação dos substratos, como relatado por Freitas et al. (2017a), Vargas; Marques (2017) e Freitas et al. (2018).

A calagem objetiva aumentar o $\mathrm{pH}$, corrigindo a acidez do solo e, consequentemente, a disponibilidade de $\mathrm{P}$, como mencionado por Silva et al. (2011) e Silva et al. (2013). Enquanto, a necessidade em $P$ está relacionada às suas funções no metabolismo da planta, como na fotossíntese, respiração, divisão e crescimento celular e, na transferência de energia como parte do trifosfato de adenosina (ATP) (DECHEN; NACHTIGALL, 2007); além do crescimento do sistema radicular (GONÇALVES et al., 2000).

Estudos têm demonstrado diferentes respostas das plantas tanto à elevação da saturação por bases quanto à adição de $\mathrm{P}$ aos substratos. Freitas et al. (2017a) verificaram que a dose de P mais adequada para a produção de mudas de Cassia grandis, na saturação de $25 \%$ foi de $600 \mathrm{mg} \mathrm{dm}^{-3}$. Para as mudas de Dalbergia nigra, Carlos et al. (2018) verificaram que a saturação por bases do substrato deve estar entre 48 e 54,7\% e a dose de $\mathrm{P}$ deve ser de $500 \mathrm{mg} \mathrm{dm}^{-3}$. Para a Plathymenia foliolosa, Freitas et al. (2017b) não verificaram a influência da calagem, porém, a dose de $\mathrm{P}$ deve ser de $300 \mathrm{mg} \mathrm{dm}^{-3}$. Ao passo que, Dutra et al. (2015) recomendaram $450 \mathrm{mg} \mathrm{dm}^{-3} \mathrm{de}$ P para mudas de Amburana cearensis.
Diante disso, o presente estudo teve por objetivo avaliar a saturação por bases e as doses de P mais favoráveis para o crescimento de mudas de ipê rosa (H. impetiginosa).

\section{Desenvolvimento}

\subsection{Metodologia}

O experimento foi realizado na casa de vegetação da Faculdade de Agronomia da Universidade de Cuiabá, campus Beira Rio I, em Cuiabá - Mato Grosso, nas coordenadas $15^{\circ} 37^{\prime} 28^{\prime}$ 'S e 56 $05^{\prime} 11^{\prime \prime}$ O. O clima predominante da região é o tropical de savana, segundo classificação de Köppen. A casa de vegetação possui um ambiente protegido com irrigação diária, porém, sem controle da temperatura.

As sementes de $H$. impetiginosa foram coletadas, ao chão, sob árvores matrizes, que foram escolhidas aleatoriamente, optando-se por árvores espaçadas em um mínimo de $100 \mathrm{~m}$ e sementes morfologicamente sadias. Essas árvores estão localizadas na Universidade Federal de Mato Grosso, campus Cuiabá, nas coordenadas $15^{\circ} 36^{\prime} 36^{\prime \prime}$ e 5 56 03'57'O. A coleta se deu, diariamente, pelo período de uma semana, em seguida, as sementes foram beneficiadas e armazenadas em ambiente refrigerado (temperatura).

Para a produção das mudas, as sementes foram colocadas para germinar em sacolas plásticas de $10 \times 15 \mathrm{~cm}$, com capacidade para $500 \mathrm{~g}$, preenchidas com substrato comercial. O substrato comercial utilizado foi o Basaplant ${ }^{\circledR}$ que possui, na sua composição, casca de pinus, fibra de coco, turfa fibrosa, vermiculita, adubação inicial com NPK e micronutrientes (sem especificação). Após 15 dias, observaram-se as primeiras germinações e, transcorridos mais 20 dias, as mudas estavam aptas para o transplante para os tratamentos testados no experimento.

O solo utilizado foi o Latossolo Vermelho distrófico com textura franco arenosa, coletado em área de Cerrado nativo do Instituto Federal de Mato Grosso, campus de São Vicente da Serra. Após coleta, uma amostra do solo foi retirada, seca ao ar, peneirada em malha de $2 \mathrm{~mm}$ e submetida às caracterizações química e física seguindo métodos descritos pela Embrapa (1997) (Quadro 1).

Quadro 1 - Análise química e física do solo

\begin{tabular}{|c|c|c|c|c|c|c|c|}
\hline $\mathrm{pH}$ & $\mathrm{K}$ & $\mathrm{P}$ & $\mathrm{H}+\mathrm{Al}$ & $\mathrm{Al}$ & $\mathrm{Ca}$ & $\mathrm{Mg}$ & SB \\
\hline $\mathrm{CaCl}_{2}$ & \multicolumn{2}{|c|}{$\mathrm{mg} \mathrm{dm}^{-3}$} & \multicolumn{5}{|c|}{$\mathrm{cmol}_{\mathrm{c}} \mathrm{dm}^{-3}$} \\
\hline 4,50 & 70,20 & 1,43 & 6,25 & 0,25 & 1,92 & 0,67 & 2,77 \\
\hline $\mathrm{T}$ & $\mathrm{t}$ & $\mathrm{V}$ & $\mathrm{m}$ & MO & Areia & Silte & Argila \\
\hline \multicolumn{2}{|c|}{$\mathrm{cmol} \mathrm{dm}^{-3}$} & \multicolumn{2}{|c|}{$\%$} & \multicolumn{4}{|c|}{$\mathrm{g} \mathrm{kg}^{-1}$} \\
\hline 9,02 & 3,02 & 30,71 & 8,28 & 34,61 & 538 & 54,30 & 407,70 \\
\hline
\end{tabular}

pH em $\mathrm{CaCl}_{2}$ - relação 1:2,5; H+Al - em acetato de cálcio; Al, Ca e Mg - em KCl 1N; P e K - em Mehlich; SB - soma de bases; T - capacidade de
troca de cátions a pH 7,0; $\mathrm{t}$ - CTC efetiva; V\% - saturação por bases, em \%; m\% - saturação por Al, em \%; MO - Matéria orgânica a partir da queima em mufla; Areia, silte e argila - método do densímetro.

Fonte: Dados da pesquisa.

O calcário foi adicionado ao solo e homogeneizado para, em seguida, ser utilizado para preencher as sacolas plásticas de
30 x $40 \mathrm{~cm}$ com capacidade para um quilo. As características do calcário estão apresentadas no Quadro 2. O cálculo da 
quantidade de calcário necessária para a elevação da saturação por bases foi realizado conforme resultados da análise de solo e o método da elevação da saturação por bases.

Quadro 2 - Características químicas e físicas do calcário utilizado

\begin{tabular}{|c|c|c|c|c|}
\hline $\mathrm{CaO}$ & $\mathrm{MgO}$ & $\mathrm{PN}$ & PRNT & $\begin{array}{c}\text { Ação } \\
\text { Residual }\end{array}$ \\
\hline 24,0 & 17,1 & 84,4 & 79 & 15,0 \\
\hline
\end{tabular}

Fonte: Dados da pesquisa.

Após o preenchimento das sacolas com solo calcareado, efetuou-se o transplante das mudas. Em seguida, as aplicações de $\mathrm{P}$, em formato de meia lua, tendo como fonte o superfosfato simples, com $18 \% \mathrm{P}_{2} \mathrm{O}_{5}, 25 \% \mathrm{CaO}$ e $12 \% \mathrm{~S}$; com base nos diferentes níveis testados no experimento. As mudas permaneceram nessas condições por 10 dias, considerandoas adaptadas após esse período, tendo sido mantidas sob irrigação diária.

O delineamento experimental utilizado foi o inteiramente casualizado, com nove tratamentos e cinco repetições: T0 - 100\% substrato comercial; T1 - V50\%; T2 - V60\%; T3 $\mathrm{V} 50 \%+50 \mathrm{~kg} \mathrm{ha}^{-1}$ de $\mathrm{P}_{2} \mathrm{O}_{5} ; \mathrm{T} 4-\mathrm{V} 50 \%+100 \mathrm{~kg} \mathrm{ha}^{-1}$ de $\mathrm{P}_{2} \mathrm{O}_{5}$; $\mathrm{T} 5-\mathrm{V} 50 \%+150 \mathrm{~kg} \mathrm{ha}^{-1}$ de $\mathrm{P}_{2} \mathrm{O}_{5} ; \mathrm{T} 6-\mathrm{V} 60 \%+50 \mathrm{~kg} \mathrm{ha}^{-1}$ de $\mathrm{P}_{2} \mathrm{O}_{5} ; \mathrm{T} 7-\mathrm{V} 60 \%+100 \mathrm{~kg} \mathrm{ha}^{-1}$ de $\mathrm{P}_{2} \mathrm{O}_{5} ; \mathrm{T} 8-\mathrm{V} 60 \%+150$ $\mathrm{kg} \mathrm{ha}^{-1}$ de $\mathrm{P}_{2} \mathrm{O}_{5}$. Após o período de pegamento, iniciou-se a análise do crescimento das mudas.

Ao final de 90 dias, a contar do pegamento das mudas, foram avaliadas as características morfológicas das plantas: altura da parte aérea $(\mathrm{H})$ com régua graduada medindo-se da base do solo até a última folha da planta; diâmetro de colo (DC) medido com paquímetro digital; e a massa seca. Para análise da massa seca, as mudas foram seccionadas em parte aérea (MSPA) e parte radicular (MSPR), levadas à estufa de circulação forçada de ar a $65^{\circ} \mathrm{C}$ até peso constante e, pesadas em balança semi-analítica. A partir da somatória dos resultados obtidos para a parte aérea e a parte radicular, obteve-se os valores para a massa seca total (MST) das mudas. Após essas análises, foi possível calcular a relação H/DC, a relação H/ MSPA e o índice de qualidade de Dickson. Para as análises dos teores de $\mathrm{N}$ nas folhas das mudas, o material foi levado ao moinho do tipo Willey e, em seguida, os teores foram obtidos mediante digestão sulfúrica e posterior destilação seguindo o método de Kjeldahl (Malavolta et al., 1997).

Os dados foram submetidos a análise de variância (ANOVA) e a comparação de médias foi realizada pelo teste de Tukey ao nível de 5\% de probabilidade de erro utilizando o programa estatístico SISVAR, após constatação da normalidade dos dados.

\subsection{Resultados}

Quanto à altura $(\mathrm{H})$, diâmetro do colo (DC) e massa seca da parte radicular (MSPR), não foi observada diferença entre os tratamentos (Quadro 3).

Quadro 3 - Altura (H) diâmetro de colo (DC), Massa Seca da Parte Aérea (MSPA), Massa Seca da Parte Radicular (MSPR), Massa Seca Total (MST) e teor de nitrogênio (N) de mudas de H. impetiginosa após crescimento em diferentes composições de substratos

\begin{tabular}{|c|c|c|c|c|c|c|}
\hline Tratamento & H (cm) & DC (mm) & MSPA (g) & MSPR (g) & MST (g) & N (g kg $\left.\mathbf{~}^{-1}\right)$ \\
\hline T0 & $39,80 \mathrm{a}$ & $4,90 \mathrm{a}$ & $2,27 \mathrm{ab}$ & $0,48 \mathrm{a}$ & $2,75 \mathrm{ab}$ & $1,90 \mathrm{~b}$ \\
\hline T1 & $42,60 \mathrm{a}$ & $4,69 \mathrm{a}$ & $1,82 \mathrm{ab}$ & $0,41 \mathrm{a}$ & $2,23 \mathrm{ab}$ & $2,18 \mathrm{~b}$ \\
\hline T2 & $43,20 \mathrm{a}$ & $4,66 \mathrm{a}$ & $1,69 \mathrm{ab}$ & $0,40 \mathrm{a}$ & $2,09 \mathrm{~b}$ & $2,63 \mathrm{ab}$ \\
\hline T3 & $45,40 \mathrm{a}$ & $4,74 \mathrm{a}$ & $1,83 \mathrm{ab}$ & $0,49 \mathrm{a}$ & $2,32 \mathrm{ab}$ & $2,63 \mathrm{ab}$ \\
\hline T4 & $42,00 \mathrm{a}$ & $5,02 \mathrm{a}$ & $1,98 \mathrm{ab}$ & $0,55 \mathrm{a}$ & $2,54 \mathrm{ab}$ & $1,96 \mathrm{~b}$ \\
\hline T5 & $42,40 \mathrm{a}$ & $4,62 \mathrm{a}$ & $1,89 \mathrm{ab}$ & $0,39 \mathrm{a}$ & $2,28 \mathrm{ab}$ & $2,68 \mathrm{ab}$ \\
\hline T6 & $40,60 \mathrm{a}$ & $4,34 \mathrm{a}$ & $1,56 \mathrm{~b}$ & $0,39 \mathrm{a}$ & $1,95 \mathrm{~b}$ & $2,80 \mathrm{ab}$ \\
\hline T7 & $44,40 \mathrm{a}$ & $4,96 \mathrm{a}$ & $2,05 \mathrm{ab}$ & $0,54 \mathrm{a}$ & $2,59 \mathrm{ab}$ & $3,75 \mathrm{a}$ \\
\hline T8 & $44,40 \mathrm{a}$ & $5,34 \mathrm{a}$ & $2,42 \mathrm{a}$ & $0,67 \mathrm{a}$ & $3,09 \mathrm{a}$ & $2,52 \mathrm{ab}$ \\
\hline CV $(\%)$ & 6,89 & 13,64 & 20,47 & 29,38 & 17,39 & 28,57 \\
\hline
\end{tabular}

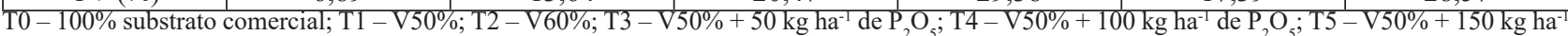
de $\mathrm{P}_{2} \mathrm{O}_{5} ; \mathrm{T} 6-\mathrm{V} 60 \%+50 \mathrm{~kg} \mathrm{ha}^{-1}$ de $\mathrm{P}_{2} \mathrm{O}_{5} ; \mathrm{T} 7-\mathrm{V} 60 \%+100 \mathrm{~kg} \mathrm{ha}^{-1}$ de $\mathrm{P}_{2} \mathrm{O}_{5} ; \mathrm{T} 8-\mathrm{V} 60 \%+150 \mathrm{~kg} \mathrm{ha}^{-1}$ de $_{2} \mathrm{O}_{5}$. Médias seguidas de mesma letra, na coluna, não diferem entre si de acordo com o teste de Tukey a $5 \%$ de probabilidade.

Fonte: Dados da pesquisa.

Para o crescimento em altura e em diâmetro, não se observou diferença entre os tratamentos com aplicação de calcário e $\mathrm{P}$, em relação ao tratamento testemunha $(100 \%$ substrato comercial). O que se observou foi que o crescimento em altura foi superior a $40 \mathrm{~cm}$ em todos aqueles com aumento da saturação por bases do solo e/ou a complementação com P, via adubação. E, o crescimento médio em diâmetro, atingiu valores acima de $5.00 \mathrm{~mm}$, nessas condições.

Os resultados podem ser um indicativo de maior adaptação da espécie a solos pouco férteis, ou de rígido ajuste da taxa de crescimento às condições de baixa disponibilidade de nutrientes, o que restringe sua resposta à melhoria nos níveis de fertilidade do solo (SIQUEIRA et al. ,1995).

Freitas et al. (2017a) não observaram resposta das mudas de Cassia grandis à aplicação de calcário e atribuíram esse resultado em função da adaptação da espécie a solos ácidos, e que os teores de $\mathrm{Ca}$ e $\mathrm{Mg}$ contidos no solo atendiam às exigências da espécie na fase inicial de crescimento. Resultados semelhantes foram observados por Gomes et al. (2004), Souza et al. (2008) e Costa Filho et al. (2013).

Xavier et al. (2009) recomendaram crescimento de 20 a 40 $\mathrm{cm}$ em altura e de $2 \mathrm{~mm}$ de diâmetro para que as mudas estejam 
aptas ao plantio no campo. Sendo assim, todos os tratamentos foram capazes de proporcionar condições favoráveis para o crescimento das mudas de $H$. impetiginosa. Vale ressaltar que, dependendo das condições nutricionais do substrato esse crescimento pode ser atingido mais rapidamente, como foi observado para os tratamentos com elevação da saturação por bases e com aplicação de adubo fosfatado.

A produção de massa seca da parte aérea (MSPA) foi influenciada positivamente pelo incremento na fertilidade do solo mediante calagem e adubação fosfatada. A maior média foi verificada no tratamento $8\left(\mathrm{~V} 60 \%+150 \mathrm{~kg} \mathrm{ha}^{-1}\right.$ de $\left.\mathrm{P}_{2} \mathrm{O}_{5}\right)$, com 2,42 g, que foi 35,5\% superior ao tratamento 6 (V60\%+ $50 \mathrm{~kg} \mathrm{ha}^{-1}$ de $\mathrm{P}_{2} \mathrm{O}_{5}$ ), um dos que apresentaram mudas com o menor desenvolvimento. O que pode estar relacionado com o baixo incremento em $\mathrm{P}$ nesse tratamento. Segundo Cruz et al. (2004) um maior peso de matéria seca de folhas é interessante para um melhor desenvolvimento das mudas, pois representa maior capacidade fotossintética e maior vigor.

Esses resultados contribuíram para a massa seca total (MST) das mudas de $H$. impetiginosa, que foi $11 \%$ superior no tratamento $8\left(\mathrm{~V} 60 \%+150 \mathrm{~kg} \mathrm{ha}^{-1}\right.$ de $\left.\mathrm{P}_{2} \mathrm{O}_{5}\right)$ em relação à testemunha. $\mathrm{O}$ que permite inferir que, o incremento no substrato proporcionou condições melhores para o desenvolvimento das mudas de $H$. impetiginosa, que se refletiu na massa seca das mudas, porém, não no crescimento em altura e em diâmetro. Resultados que já são favoráveis porque, o desenvolvimento da massa foliar favoreceu a capacidade fotossintética da planta. Embora não se tenha observado diferença entre os tratamentos para a produção de massa seca da parte radicular (MSPR), mesmo essa característica apresentando média $28,3 \%$ superior no tratamento 8 , em relação à testemunha.

De acordo com Gomes; Paiva (2012) o adequado suprimento em $\mathrm{P}$ no início do crescimento da planta é importante para a formação dos primórdios vegetativos, uma vez que as raízes de plantas jovens absorveram fosfato mais rapidamente que raízes de plantas mais velhas.

Macedo e Teixeira (2012) não observaram diferença da calagem e da adubação fosfatada no crescimento da Eugenia stipitata e atribuíram esses resultados ao fato de que, o adubo foi aplicado de forma localizada e superficialmente, dessa forma, não seria necessário um maior investimento em crescimento radicular pelas mudas para ter acesso a uma concentração mais alta de $\mathrm{P}$ e, consequentemente, para a absorção de P.

Os teores de $\mathrm{N}$ não influenciaram no crescimento das mudas de $H$. impetiginosa, apesar de serem observadas médias inferiores a $4 \mathrm{~g} \mathrm{~kg}^{-1}$ de $\mathrm{N}$ em todos os tratamentos testados. Nesse caso, a maior média foi verificada no tratamento $7\left(\mathrm{~V} 60 \%+100 \mathrm{~kg} \mathrm{ha}^{-1}\right.$ de $\left.\mathrm{P}_{2} \mathrm{O}_{5}\right)$, porém, esse não foi o que apresentou os melhores resultados e características de crescimento das mudas indicando que, esses teores de $\mathrm{N}$ não influenciaram no crescimento e/ou na produção de massa seca, podendo esta espécie de ipê, ser uma espécie adaptada a condições de solos com baixos teores de N.

Ao analisar as relações e o índice de qualidade de Dickson (Quadro 4), verificou-se diferença apenas para o índice de qualidade, que apresentou média superior no tratamento 8. Provavelmente, em função da produção de massa seca observada nesse tratamento.

Quadro 4 - Relação altura/diâmetro de colo (H/D), relação massa seca da parte aérea/massa seca da parte radicular (MSPA/MSPR) e índice de qualidade de Dickson de mudas de H. impetiginosa após crescimento em diferentes composições de substratos

\begin{tabular}{|c|c|c|c|}
\hline Tratamento & H/DC & MSPA/MSPR & DICKSON \\
\hline T0 & $8,44 \mathrm{a}$ & $5,76 \mathrm{a}$ & $0,21 \mathrm{ab}$ \\
\hline $\mathrm{T} 1$ & $9,11 \mathrm{a}$ & $5,13 \mathrm{a}$ & $0,16 \mathrm{ab}$ \\
\hline $\mathrm{T} 2$ & $9,52 \mathrm{a}$ & $4,57 \mathrm{a}$ & $0,16 \mathrm{ab}$ \\
\hline $\mathrm{T} 3$ & $9,83 \mathrm{a}$ & $3,77 \mathrm{a}$ & $0,17 \mathrm{ab}$ \\
\hline $\mathrm{T} 4$ & $8,43 \mathrm{a}$ & $3,69 \mathrm{a}$ & $0,21 \mathrm{ab}$ \\
\hline $\mathrm{T} 5$ & $9,22 \mathrm{a}$ & $4,81 \mathrm{a}$ & $0,16 \mathrm{ab}$ \\
\hline $\mathrm{T} 6$ & $9,40 \mathrm{a}$ & $4,08 \mathrm{a}$ & $0,15 \mathrm{~b}$ \\
\hline $\mathrm{T} 7$ & $8,95 \mathrm{a}$ & $3,93 \mathrm{a}$ & $0,20 \mathrm{ab}$ \\
\hline $\mathrm{T} 8$ & $8,38 \mathrm{a}$ & $3,84 \mathrm{a}$ & $0,25 \mathrm{a}$ \\
\hline $\mathrm{CV}(\%)$ & 14,22 & 36,22 & 24,88 \\
\hline
\end{tabular}

T0 - $100 \%$ substrato comercial; T1 - V50\%; T2 - V60\%; T3 - V50\% + $50 \mathrm{~kg} \mathrm{ha}^{-1}$ de $\mathrm{P}_{2} \mathrm{O}_{5} ; \mathrm{T} 4-\mathrm{V} 50 \%+100 \mathrm{~kg} \mathrm{ha}^{-1}$ de $\mathrm{P}_{2} \mathrm{O}_{5} ; \mathrm{T} 5-\mathrm{V} 50 \%+150$ $\mathrm{kg} \mathrm{ha}^{-1}$ de $\mathrm{P}_{2} \mathrm{O}_{5} ; \mathrm{T} 6-\mathrm{V} 60 \%+50 \mathrm{~kg} \mathrm{ha}^{-1} \mathrm{de}_{2} \mathrm{O}_{5} ; \mathrm{T} 7-\mathrm{V} 60 \%+100 \mathrm{~kg} \mathrm{ha}^{-1}$ de $\mathrm{P}_{2} \mathrm{O}_{5} ; \mathrm{T} 8-\mathrm{V} 60 \%+150 \mathrm{~kg} \mathrm{ha}^{-1}$ de $\mathrm{P}_{2} \mathrm{O}_{5}$. Médias seguidas de mesma letra, em coluna, não diferem entre si de acordo com o teste de Tukey a $5 \%$ de probabilidade.

Fonte: Dados da pesquisa.

Segundo Moreira e Moreira (1996), a relação H/D indica o padrão de qualidade das mudas, que, de acordo com Birchler et al. (1998) deve ser menor que 10. Característica que foi observada em todos os tratamentos testados. Enquanto, para o índice de qualidade de Dickson, o valor obtido deve ser de, no mínimo, 0,20 (Hunt, 1990), que foi observado nos tratamentos testemunha, T4 (V50\% + $100 \mathrm{~kg} \mathrm{ha}^{-1}$ de $\left.\mathrm{P}_{2} \mathrm{O}_{5}\right)$, T7 (V60\% $+100 \mathrm{~kg} \mathrm{ha}^{-1}$ de $\left.\mathrm{P}_{2} \mathrm{O}_{5}\right)$ e T8 $\left(\mathrm{V} 60 \%+150 \mathrm{~kg} \mathrm{ha}^{-1}\right.$ de $\left.\mathrm{P}_{2} \mathrm{O}_{5}\right)$. Sendo que, em T4, T7 e T8, houve o incremento mínimo de $100 \mathrm{~kg} \mathrm{ha}^{-1}$ de $\mathrm{P}_{2} \mathrm{O}_{5}$, corroborando com o que se verificou para as demais características que, as adições de calcário e de $\mathrm{P}$ melhoraram as condições para o desenvolvimento das mudas de H. impetiginosa. No entanto, Gomes e Paiva (2011) recomendaram que este valor seja o maior possível, pois, quanto maior, melhor será o padrão de qualidade das mudas.

\section{Conclusão}

A saturação por bases e a adubação fosfatada não influenciam o crescimento em altura e em diâmetro das mudas de $H$. impetiginosa, porém, influenciam na produção de massa seca. Para a produção de mudas de $H$. impetiginosa recomenda-se a utilização de solo em melhores condições de fertilidade, sendo esta atingida com $\mathrm{V} 60 \%+150 \mathrm{~kg} \mathrm{ha}^{-1} \mathrm{de}$ $\mathrm{P}_{2} \mathrm{O}_{5}$.

\section{Referências}

ALVAREZ, V.H. Equilibrio de formas disponíveis de fósforo e enxofres em dois latossolos de Minas Gerais. Viçosa: 
Universidade Federal de Viçosa, 1974.

BIRCHLER, T. et al. La planta ideal: revision del concepto, parametros definitorios e implementation practica. Invest. Agr., v.7, n. 1/2, p.109-121, 1998 .

CARLOS, L. et al. Liming and Phosphating in Dalbergia nigra (Vell.) Allemão ex Benth. seedlings. Flor. e Amb., v.25, n.4, p.110, 2018. doi:10.1590/2179-8087.023917.

COSTA FILHO, R.T.; VALERI, S.V.; CRUZ, M.C.P. Calagem e adubação fosfatada no crescimento de mudas de Mimosa caesalpinifolia Benth. em Latossolo vermelho-amarelo. Ciênc. Flor, v.23, n.1, p.89-98. 2013. doi:10.5902/198050988442.

CRUZ, C.A.F. et al. Efeito de diferentes níveis de saturação por bases no desenvolvimento e qualidade de mudas de ipê-roxo (Tabebuia impetiginosa (Mart.) Standley). Scei. For., n.66, p.100107, 2004.

DECHEN, A.R.; NACHTIGALL, G.R. Elementos requeridos à nutrição de plantas. In: NOVAIS, R. F. et al. Fertilidade do solo. Viçosa, MG: SBCS, 2007. p.91-132.

DUTRA, T.R. et al. Crescimento de mudas de umburana (Amburana cearensis) em resposta à adubação com nitrogênio e fósforo. Agrop. Cient. no Sem., v.11, n.4, p.42-52, 2015. doi:10.30969/acsa.v11i4.694.

EMBRAPA. Centro Nacional de Pesquisa de Solos. Manual de métodos de análise de solos. 2. ed. Rio de Janeiro: Embrapa, 1997.

FREITAS, E.C.S. et al. Crescimento e qualidade de mudas de Cassia grandis Linnaeus f. em resposta à adubação fosfatada e calagem. Ciênc. Flor., v.27, n.2, p.509-519, 2017a. doi: 10.5902/1980509827732.

FREITAS, E.C.S. et al. Effect of phosphate fertilization and base saturation of substrate on the seedlings growth and quality of Plathymenia foliolosa Benth. Rev. Arv., v.41, n.1, p.1-9, $2017 \mathrm{~b}$. doi: 10.1590/1806-90882017000100011.

FREITAS, E.C.S. et al. Crescimento de mudas de Dipteryx alata sob adubação fosfatada e calagem. Amb., v.14, n.2, p.267-281, 2018. doi: 10.5935/ambiencia.2018.02.05

GEMAQUE, R.C.R.; DAVIDE, A.C.; FARIA, J.M.R. Indicadores de maturidade fisiológica de sementes de ipê-roxo (Tabebuia impetiginosa (Mart.) Standl.). Rev. Cer., v.8, n.2, p.84-91, 2002.

GOMES, K.C.O. et al. Influência da saturação por bases e do fósforo no crescimento de mudas de angico-branco. Rev. Árv., Viçosa, v.28, n.6, p.785-792, 2004.

GOMES, J.M.; PAIVA, H.N. Viveiros florestais. Viçosa: Editora
UFV, 2011.

GONÇALVES, J.L.M. et al. Produção de mudas de espécies nativas: substrato, nutrição, sombreamento e fertilização. In: GONÇALVES, J. L. M.; BENEDETTI, V. Nutrição e fertilização florestal. Piracicaba: IPEF, 2000. p.309-350.

HUNT, G.A. Effect of styroblockn design and cooper treatment on morphology of conifer seedlings. In: Target seedlings Symposium, meeting of the western forest nursery associations, General technical report RM-200, 1990, Roseburg, p.218-222.

MACEDO, S.T.; TEIXEIRA, P.C. Calagem e adubação fosfatada para formação de mudas de araçá-boi. Act. Amaz., v.42, n.3, p.405-412, 2012. doi:10.1590/S0044-59672012000300013.

MALAVOLTA, E.; VITTI, G.C.; OLIVEIRA, S.A. Avaliação do estado nutricional das plantas: princípios e aplicações. Piracicaba: POTAFOS, 1997.

MOREIRA, F.M.S.; MOREIRA, F.W. Característica de germinação de 64 espécies de leguminosas florestais nativas da Amazônia, em condições de viveiro. Act. Amaz., v.26, n.1/2, p.316, 1996. doi:10.1590/1809-43921996261016.

PASSOS, M.A.A. Efeito da calagem e de fósforo no crescimento inicial da algaroba (Prosopis juliflora (SW) DC). Viçosa: Universidade Federal de Viçosa, 1994.

SILVA, E.A.A. et al. Germination studies on Tabebuia impetiginosa Mart. seeds. Rev. Cer., v.10, n.1, p.1-9, 2004.

SILVA, T.A.F. et al. Calagem e adubação fosfatada para a produção de mudas de Swietenia macrophylla. Rev. Flor., v.41, n.3, p.459-470. 2011. doi:10.5380/rf.v41i3.23992.

SILVA, P.M.C. et al. Efeito do potássio e do calcário na qualidade de mudas de cedro doce (Bombacopsis quinata). Rev. Agroamb., v.7, n.1, p.63-69. 2013. doi:10.18227/1982-8470ragro.v7i1.842.

SIQUEIRA, J.O. et al. Aspectos de solos, nutrição vegetal e microbiologia na implantação de matas ciliares. Belo Horizonte: CEMIG, 1995.

SOUZA, P.H. et al. Influência da saturação por bases do substrato no crescimento e qualidade de mudas de Machaerium nictitans (Vell.) Benth. Rev. Arv., v.32, n.2, p.193-201, 2008. doi:10.1590/ S0100-67622008000200001.

VARGAS, G.; MARQUES, R. Crescimento e nutrição de angico e canafístula sob calagem e gessagem. Rev. Flor., v.24, p.1-10, 2017. doi: 10.1590/2179-8087.010216.

XAVIER, A.; WENDLING, I.; SILVA, R.L. Silvicultura clonal: princípios e técnicas. Viçosa: UFV, 2009. 